\title{
Energetics of $\mathrm{Co}$ adatoms on the $\mathrm{Cu}(001)$ surface
}

\author{
N. A. Levanov, V. S. Stepanyuk, * and W. Hergert \\ Fachbereich Physik, Martin-Luther-Universität, Fr.-Bach-Platz 6, D-06099 Halle, Germany \\ D. I. Bazhanov \\ Max-Planck-Institute für Mikrostrukturphysik, Weinberg 2, 06120 Halle, Germany \\ and Solid State Physics Department, Moscow State University, 119899 Moscow, Russia \\ P. H. Dederichs \\ Institut für Festkörperforschung, Forschungszentrum Jülich, D-52425 Jülich, Germany
}

A. Katsnelson

Solid State Physics Department, Moscow State University, 119899 Moscow, Russia

C. Massobrio

Institut de Physique et de Chimie des Matériaux de Strasbourg, 23 rue du Loess F-67037 Strasbourg, France

(Received 13 April 1999; revised manuscript received 26 July 1999)

\begin{abstract}
By using an $\mathrm{N}$-body potential scheme constructed by fitting the interaction parameters to accurate firstprinciples calculations, we investigate the structural stability of Co atoms and clusters deposited on $\mathrm{Cu}(100)$. We found that Co atoms and clusters prefer to be embedded inside the substrate, in a way compatible with the formation of a surface alloy observed experimentally. Enhanced stability is achieved when Co atoms are deposited on a preformed Co cluster embedded on the uppermost layer of the substrate. Co atoms deposited on Co islands are best stabilized when they concur to complete the islands, by promoting layer-by-layer growth.
\end{abstract}

Ultrathin films of ferromagnetic metals have found considerable interest in recent years due to their technological applications in the area of magneto-optical and transport properties. ${ }^{1-3}$ In particular the growth of Fe and Co films on $\mathrm{Cu}(001)$, which takes place pseudomorphically on the fcc substrate, has been investigated extensively. ${ }^{4-10}$ The quality of the grown layers and of the interfaces has a strong influence on properties like giant magnetoresistance, ${ }^{5}$ magnetic anisotropy, ${ }^{6,7}$ and oscillatory interlayer exchange coupling. ${ }^{8,9}$ Kief and Egelhoff ${ }^{10}$ have reported the observation of nonideal film growth, characterized by the formation of compact $\mathrm{Co}$ clusters and the segregation of substituted $\mathrm{Cu}$ on the surface. Recently, the interfacial intermixing of ultrathin Co films on a $\mathrm{Cu}(001)$ was observed, ${ }^{11}$ despite the fact that $\mathrm{Co}$ and $\mathrm{Cu}$ are immiscible in the bulk. ${ }^{12}$ The intermixing in the upper layers might not only be favored kinetically, but also energetically. ${ }^{13}$

In this paper we resort to a newly developed $n$-body interatomic potential scheme to ascertain the energetics of atoms and clusters of $\mathrm{Co}$ on the $\mathrm{Cu}(001)$. A strong tendency for a direct exchange mechanism into the $\mathrm{Cu}$ layer is found. Our results demonstrate that at the initial stage of monolayer growth small Co clusters are formed in the $\mathrm{Cu}$ surface. We investigate the mechanism of adatom-cluster interactions and show how heteroepitaxial thin film growth takes place.

Our approach is based on accurate first-principles calculations of selected cluster-substrate properties, which have been employed in the fitting of the potential parameters. This results in a manageable and inexpensive scheme able to account for structural relaxation and including implicitly mag- netic effects, crucial for a realistic determination of interatomic interactions in systems having a magnetic nature.

The potentials are formulated in the second moment tightbinding approximation (TB-SMA). ${ }^{14,15}$ The attractive term (band energy) $E_{B}^{i}$ contains the many-body interaction. The repulsive term $E_{R}^{i}$ is described by pair interactions (BornMayer form). The cohesive energy $E_{c o h}$ is the sum of the band energy and repulsive part:

$$
\begin{gathered}
E_{c o h}=\sum_{i}\left(E_{R}^{i}+E_{B}^{i}\right), \\
E_{R}^{i}=\sum_{j} A_{\alpha \beta} \exp \left[-p_{\alpha \beta}\left(\frac{r_{i j}}{r_{0}^{\alpha \beta}}-1\right)\right], \\
E_{B}^{i}=-\left\{\sum_{j} \xi_{\alpha \beta}^{2} \exp \left[-2 q_{\alpha \beta}\left(\frac{r_{i j}}{r_{0}^{\alpha \beta}}-1\right)\right]\right\}^{1 / 2} .
\end{gathered}
$$

$r_{i j}$ is the distance between the atoms $i$ and $j . r_{0}^{\alpha \beta}$ is the first neighbor distance in the crystalline structures of the pure metals for atom-like interactions and becomes an adjustable parameter in the case of the cross interaction. $\xi$ is an effective hopping integral; $p_{\alpha \beta}$ and $q_{\alpha \beta}$ describe the decay of the interaction strength with distance of the atoms.

After determination of the $\mathrm{Cu}-\mathrm{Cu}$ parameters which are fitted to experimental data only (see Ref. 14 and Ref. 15), the $\mathrm{Co}-\mathrm{Co}$ and $\mathrm{Co}-\mathrm{Cu}$ parameters are optimized simultaneously by including in the fit the results of first-principles KorringaKohn-Rostoker (KKR) calculations. ${ }^{16}$ To this purpose, we 
TABLE I. Data used for the fitting of the potential together with the values calculated with the optimized potential. (Cohesive energy $E_{c}$, bulk modulus $B$, elastic constants $C_{i j}$ from Refs. 14,15, first and second neighbor interaction energies $E_{1, b}^{\mathrm{Co}-\mathrm{Co}}, E_{2, b}^{\mathrm{Co}-\mathrm{Co}}$ from Ref. 17, solution energy $E_{S}^{\mathrm{Co}}$ in $\mathrm{Cu}$ from Ref. 18, binding energies of small Co clusters $E_{1, \text { on } \mathrm{Cu}(100)}^{\mathrm{Co}-\mathrm{Co}}, E_{1, \text { in } \mathrm{Cu}(100)}^{\mathrm{Co}-\mathrm{Co}}, E_{\text {on } \mathrm{Cu}(100)}^{\text {trimer }}, E_{\text {on } \mathrm{Cu}(100)}^{2 \times 2 \text { island }}$ are calculated using the KKR Green's function method.)

\begin{tabular}{|c|c|c|c|}
\hline & Quantity & Data & Fitted value \\
\hline $\mathrm{Cu}$ & $a_{C u}$ & $3.615 \AA$ & $3.614 \AA$ \\
\hline \multirow[t]{5}{*}{ (fcc) } & $E_{c}$ & $3.544 \mathrm{eV}$ & $3.545 \mathrm{eV}$ \\
\hline & $B$ & 1.42 Mbar & 1.42 Mbar \\
\hline & $C_{11}$ & 1.76 Mbar & 1.76 Mbar \\
\hline & $C_{12}$ & 1.25 Mbar & 1.25 Mbar \\
\hline & $C_{44}$ & $0.82 \mathrm{Mbar}$ & $0.82 \mathrm{Mbar}$ \\
\hline $\mathrm{Co}$ & $a_{\mathrm{Co}}$ & $2.507 \AA$ & $2.515 \AA$ \\
\hline \multirow[t]{7}{*}{ (hcp) } & $E_{c}(\mathrm{E})$ & $4.386 \mathrm{eV}$ & $4.395 \mathrm{eV}$ \\
\hline & B & 1.948 Mbar & 1.989 Mbar \\
\hline & $C_{11}$ & 3.195 Mbar & 3.337 Mbar \\
\hline & $C_{12}$ & 1.661 Mbar & 1.426 Mbar \\
\hline & $C_{13}$ & 1.021 Mbar & 1.178 Mbar \\
\hline & $C_{33}$ & 3.736 Mbar & 3.665 Mbar \\
\hline & $C_{44}$ & $0.824 \mathrm{Mbar}$ & 0.646 Mbar \\
\hline \multirow[t]{7}{*}{$\mathrm{Co}-\mathrm{Cu}$} & $E_{S}^{\mathrm{Co} \text { in } \mathrm{Cu}}$ & $0.4 \mathrm{eV}$ & $0.38 \mathrm{eV}$ \\
\hline & $E_{1, b}^{\mathrm{Co}-\mathrm{Co}}$ & $-0.12 \mathrm{eV}$ & $-0.18 \mathrm{eV}$ \\
\hline & $E_{2, b}^{\mathrm{Co}-\mathrm{Co}}$ & $0.03 \mathrm{eV}$ & $-0.05 \mathrm{eV}$ \\
\hline & $E_{1, \text { on }}^{\mathrm{Co} C \mathrm{Cu}(100)}$ & $-1.04 \mathrm{eV}$ & $-1.04 \mathrm{eV}$ \\
\hline & $E_{1, \text { in }}^{\mathrm{Co} C u(100)}$ & $-0.26 \mathrm{eV}$ & $-0.35 \mathrm{eV}$ \\
\hline & $E_{o n ~}^{\text {trimer }} \mathrm{Cu}(100)$ & $-2.06 \mathrm{eV}$ & $-1.96 \mathrm{eV}$ \\
\hline & $E_{\text {on } \mathrm{Cu}(100)}^{2 \times 2 \text { island }}$ & $-3.84 \mathrm{eV}$ & $-3.86 \mathrm{eV}$ \\
\hline
\end{tabular}

have taken the solution energy of a single Co impurity in bulk Cu, $E_{S}^{\mathrm{Co} \text { in } \mathrm{Cu}}$ (Ref. 17), energies of interaction of two Co impurities in $\mathrm{Cu}$ bulk, $E_{1, b}^{\mathrm{Co}-\mathrm{Co}}, E_{2, b}^{\mathrm{Co}-\mathrm{Co}}$ (Ref. 18), and binding energies of small supported Co clusters on $\mathrm{Cu}(001)$, $E_{1, \text { on } \mathrm{Cu}(100)}^{\mathrm{Co}-\mathrm{Co}}, E_{1, \text { in } \mathrm{Cu}(100)}^{\mathrm{Co}-\mathrm{Co}}$ (terrace position), $E_{\text {on } \mathrm{Cu}(100)}^{\text {trimer }}$, $E_{\text {on }}^{2 \times 2} \mathrm{Cu}(100)$. Calculations for clusters on metal surfaces are based on density functional theory in the local spin density approximation and KKR Green's function method for lowdimensional systems. ${ }^{16}$ We treat the ideal surface as a twodimensional perturbation of the bulk. The Green's function of the ideal surface and the Green's function of clusters on the surface are calculated using the multiple-scattering theory. Details of the method can be found elsewhere. ${ }^{16}$

The simultaneous determination of the $\mathrm{Cu}-\mathrm{Cu}, \mathrm{Co}-\mathrm{Co}$, and $\mathrm{Cu}-\mathrm{Co}$ interaction potentials for the study of surface and interface properties needs more flexibility than the standard form of TB-SMA is able to provide. Therefore a modified form of the repulsive part is used:

$$
E_{R}^{i}=\sum_{j}\left[A_{\alpha \beta}^{1}\left(\frac{r_{i j}}{r_{0}^{\alpha \beta}}-1\right)+A_{\alpha \beta}^{0}\right] \exp \left[-p_{\alpha \beta}\left(\frac{r_{i j}}{r_{0}^{\alpha \beta}}-1\right)\right]
$$

The standard form of the repulsive potential $\left(A_{\alpha \beta}^{1}=0\right)$ is used for the $\mathrm{Cu}-\mathrm{Cu}$ interaction. The modified form of Eq. (4) is used for the $\mathrm{Co}-\mathrm{Co}$ and $\mathrm{Cu}-\mathrm{Co}$ interactions.

The set of data used to define the potential and the corresponding values calculated by means of the optimized poten-
TABLE II. Parameters of interatomic interactions.

\begin{tabular}{lccc}
\hline \hline Parameter & $\mathrm{Cu}-\mathrm{Cu}$ & $\mathrm{Co}-\mathrm{Co}$ & $\mathrm{Co}-\mathrm{Cu}$ \\
\hline$A^{1}(\mathrm{eV})$ & 0.0 & -0.852 & -1.905 \\
$A^{0}(\mathrm{eV})$ & 0.086 & 0.139 & -0.049 \\
$\xi(\mathrm{eV})$ & 1.2240 & 1.5247 & 0.7356 \\
$p$ & 10.96 & 7.679 & 8.183 \\
$q$ & 2.278 & 2.139 & 3.344 \\
$r_{0}(\AA)$ & 2.556 & 2.378 & 2.405 \\
\hline \hline
\end{tabular}

tial are given in Table I. The bulk and surface properties are well reproduced. The parameters of interatomic interactions are presented in Table II.

It is important to note that the optimum length of the $\mathrm{Co}-\mathrm{Cu}$ bonds are strongly influenced by the misfit strain. The data used for the fitting of the $\mathrm{Co}-\mathrm{Cu}$ potentials do not include the effect of relaxation of the lattice around Co impurities. Therefore it is necessary to test our potentials performing calculations of relaxations near $\mathrm{Co}$ in the $\mathrm{Cu}$ bulk and on the $\mathrm{Cu}$ surface. We investigate the lattice distortion in the vicinity of the Co impurity and compare present results with a first-principles study of lattice relaxation by means of the KKR Green's function method recently performed in our group. ${ }^{19}$ Semiempirical and $a b$ initio studies show that the $\mathrm{Cu}$ lattice is compressed due to the Co impurities. A change of the first nearest neighbor distance near the Co impurity, found in the present calculation, is $\approx 1 \%$, while the firstprinciples calculation gives $\approx 0.5 \%$. The second test of our potential is done performing calculations of interlayer distances in $\mathrm{Co} / \mathrm{Cu}$ multilayers. A detailed low-energy electron diffraction study of the $\mathrm{Co} / \mathrm{Cu}(100)$ films for different $\mathrm{Co}$ coverages was performed in Kirschner's group. ${ }^{20}$ Interlayer spacing was determined for different Co coverages. We use our potentials to determine interlayer distances performing energy minimization calculations. Results presented in Table III demonstrate that the agreement with experiment is rather good. Therefore we believe that the parametrization developed in our paper gives a good description of the $\mathrm{Co}-\mathrm{Cu}$ bonds.

The energetics of the elementary exchange process is considered first. In our total energy calculations the replacement of a $\mathrm{Cu}$ atom by the deposited $\mathrm{Co}$ atom [Fig. 1(b)] is preferred by $0.50 \mathrm{eV}$ to its adsorption on the $\mathrm{Cu}(001)$ substrate [Fig. 1(a)]. Hereafter this exchange process will be referred to as "direct", exchange. The same value calculated without relaxation was found to be $0.45 \mathrm{eV}$. This value is in good agreement with our calculations by means of the KKR

TABLE III. Interlayer distances for $\mathrm{Co} / \mathrm{Cu}(001)$. The experimental values are taken from Ref. 20. $d_{i j}$ denotes the spacing (in angstroms) between deposited Co monolayers (ML) $i$ and $j$, starting from the $\mathrm{Cu}$ surface layer with index 0 .

\begin{tabular}{lcccccc}
\hline \hline & \multicolumn{2}{c}{$1 \mathrm{ML}$} & \multicolumn{2}{c}{$2 \mathrm{ML}$} & \multicolumn{2}{c}{$3 \mathrm{ML}$} \\
& $\mathrm{MD}$ & Expt. & $\mathrm{MD}$ & Expt. & $\mathrm{MD}$ & Expt. \\
\hline$d_{01}$ & 1.77 & \multirow{2}{*}{$1.78 \pm 0.03$} & 1.76 & $1.73 \pm 0.03$ & 1.76 & $1.76 \pm 0.03$ \\
$d_{12}$ & & & 1.71 & $1.77 \pm 0.02$ & 1.70 & $1.74 \pm 0.02$ \\
$d_{23}$ & & & & & 1.72 & $1.76 \pm 0.02$ \\
\hline \hline
\end{tabular}



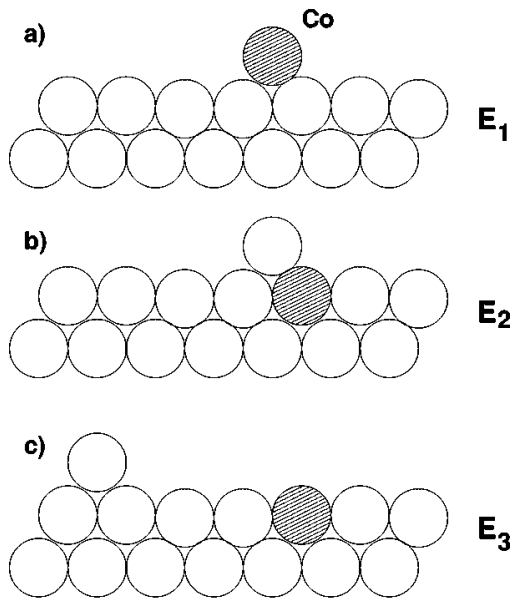

\section{$E_{2}-E_{1}=-0.50 \mathrm{eV}(-0.45 \mathrm{eV})$ $E_{3}-E_{1}=-0.41 \mathrm{eV}(-0.38 \mathrm{eV})$}

FIG. 1. Energetics of the exchange of $\mathrm{Co}$ and $\mathrm{Cu}$ at the $\mathrm{Cu}(100)$ surface. Energies calculated without relaxation are given in brackets.

Green's function method (0.54 eV, without relaxation). When the energy difference with respect to the configuration depicted in Fig. 1(a) is taken by considering a much larger distance between the $\mathrm{Co}$ atom and the promoted $\mathrm{Cu}$ atom on the substrate [Fig. 1(c)], we obtain $-0.41 \mathrm{eV}$. The corresponding value obtained without relaxation is $-0.38 \mathrm{eV}$. We call this process a "complete", exchange. These calculations suggest that surface alloying is energetically favorable in the case of $\mathrm{Co} / \mathrm{Cu}(001)$, a result essentially unmodified by the inclusion of structural relaxations. In addition, we calculated that the gain of energy by the transfer of a Co atom from the topmost to the next layer of the $\mathrm{Cu}(001)$ substrate is $-0.59 \mathrm{eV}$. The energy gain by a further movement of Co into the substrate is smaller than $0.1 \mathrm{eV}$ and, as expected, vanishes for a further movement of Co into the bulk. ${ }^{21} \mathrm{We}$ recall that the exchange process for $3 d$ impurities on $\mathrm{Fe}(001)$ has been recently investigated on the basis of total energy calculations via the KKR Green's function method. ${ }^{22}$ Unlike in the present case, it was found that for all $3 d$ impurities the complete exchange configuration is more stable than the direct exchange. This different behavior is likely to be related to the different magnetic nature of the substrate.

The total energy difference between the dimer complex and two isolated impurities at infinite separation (see Fig. 2) can be considered as the effective interaction energy between the two impurities. Co atoms embedded in the first layer prefer to form clusters, as proved by the energy gained $(-0.38 \mathrm{eV})$, when two Co atoms, originally far apart within the layer, are moved together in the nearest neighbor configuration. The interaction energy for two Co impurities on next nearest neighbor sites in the topmost layer is $-0.12 \mathrm{eV}$. For the second and deeper layers the energy gain due to the aggregation of two Co impurities to the positions of nearest neighbors is equal to $-0.19 \mathrm{eV}$. The corresponding value for next nearest neighbors is $-0.09 \mathrm{eV}$. We have found in our calculations that the Co dimer complex gains an energy up to $1 \mathrm{eV}$ in the second surface layer compared to the first surface one.
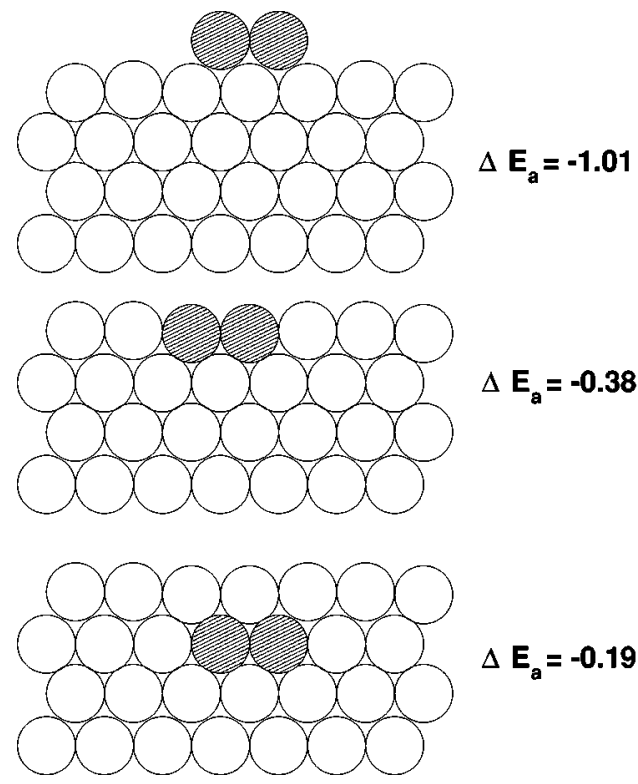

FIG. 2. Energy gain due to Co pair formation in different layers of the $\mathrm{Cu}(001)$ substrate.

One can conclude that two Co atoms attract each other in the topmost as well as in deeper layers of the substrate and, as a consequence, Co impurities should form clusters in top layers on the $\mathrm{Cu}(001)$ substrate. Indeed, formation of $\mathrm{Co}$ and Fe clusters in $\mathrm{Cu}(001)$ was observed experimentally by Fassbender et al. ${ }^{11}$ and Johnson et al. ${ }^{2}$ using scanning-tunneling microscopy techniques.

Let us now consider adatom-cluster interactions. It is shown in Fig. 3(a) that adsorbed Co atoms prefer to sit on top of the cluster formed by the embedded Co atoms. The adsorption energy for Co adatoms far from the cluster on the $\mathrm{Cu}(001)$ substrate $(2.36 \mathrm{eV})$ is much lower than the one calculated for Co adatoms on top of the embedded Co cluster $(3.68 \mathrm{eV})$. Accordingly, at the initial stages of thin film growth, Co islands in the topmost layer of the substrate could be considered as pinning centers for further adsorption of $\mathrm{Co}$ atoms. Two possible growth modes for Co clusters on the $\mathrm{Cu}(001)$ surface can then be envisaged. On the one hand, the
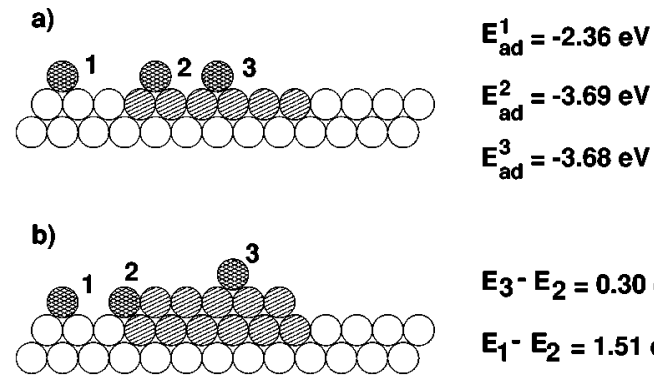

$E_{3}-E_{2}=0.30 \mathrm{eV}$

$E_{1}-E_{2}=1.51 \mathrm{eV}$

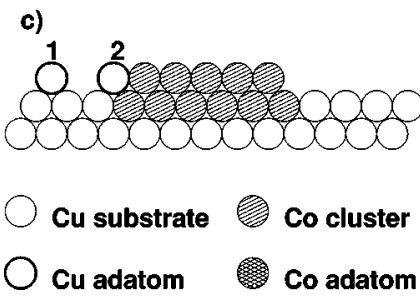

$E_{2}-E_{1}=-0.37 \mathrm{eV}$

FIG. 3. Energetics of the cluster formation at the surface. 
growth of three-dimensional clusters will take place provided the deposition energy of Co atoms is small. On the other hand, one can expect that if the kinetic energy of the deposited adatoms is large, the atoms can migrate from the top of the cluster down to the adsorption sites on the first layer, so as to enlarge the cluster and promote layer-by-layer growth. We focus here on the energetics of this process.

By starting from a preformed small Co cluster, incoming Co atoms can take one of the following three positions [Fig. 3(b)]: (i) far away from the cluster on the surface (pos. 1), (ii) near the edge of the cluster (pos. 2), and (iii) on the top of the cluster (pos. 3). The configuration with the adsorbed Co adatom near the edge of the Co cluster was found to be the most stable one. This means that it is favorable for a Co atom to jump from the top of the island to the uppermost layer level and reside near the edge of the cluster. The energy gain corresponding to this configuration is $0.30 \mathrm{eV}$. The above process can occur provided the fraction of the atomic kinetic energy still available after transfer and dissipation into the substrate is sufficiently large to overcome the Schwoebel barrier. ${ }^{23}$ On the basis of our calculations we also observe that $\mathrm{Co}$ atoms adsorbed on the surface are more stable when they reach the edge of the cluster, as indicated by the large energy gained $(-1.51 \mathrm{eV})$ for such a process.

Finally, let us consider the situation in which additional $\mathrm{Cu}$ atoms have been promoted by the exchange on the top of the substrate [Fig. 3(c)]. When a $\mathrm{Cu}$ atom approaches the cluster to stick to its edge, the energy of the system is lowered by $0.37 \mathrm{eV}$. Therefore islands will continue to grow by incorporating either $\mathrm{Cu}$ or $\mathrm{Co}$.

In summary we have investigated the energetics of an heterogenous system consisting of Co atoms either adsorbed on the top of a $\mathrm{Cu}(100)$ substrate or embedded within it. On the experimental side, it appears that a surface alloy might be formed upon deposition of $\mathrm{Co}$ on $\mathrm{Cu}(001)$. We have addressed this issue by relying on a sound theoretical model able to conjugate (a) a careful determination of the interatomic forces which involves magnetic contributions and (b) a flexible account of relaxation effects. This goal has been achieved by constructing an $n$-body interatomic potential partially fitted to accurate $a b$ initio data. This tool has allowed us to draw the following conclusions. First, Co atoms lower the energy of the total system when they lie inside the $\mathrm{Cu}(100)$ substrate, more then when they are adsorbed on the top of it. The $\mathrm{Co} / \mathrm{Cu}(100)$ system is further stabilized when the Co atoms form clusters, and when these clusters reside in deeper layer of the substrate. The most recent experiments performed by Zimmermann et al. ${ }^{24}$ have found a burrowing of $\mathrm{Co}$ nanoparticles in $\mathrm{Cu}$ substrate. This finding is in line with our main results.

We have found that the adsorption energy of a single Co atom is much higher when the deposition takes place above a preformed Co cluster, embedded on the first layer of the substrate. This gives rise to special sites on the surface terraces acting as preferential centers for growth. We have also proved that layer-by-layer growth is preferred from the energetic point of view, since Co atoms prefer to join an existing adsorbed Co cluster. We would like to emphasize that the main results of our calculations show that atoms try to maximize their total number of neighbors in both Co-Co and $\mathrm{Co}-\mathrm{Cu}$ arrangements.

The considerations developed in this paper do not account for kinetic effects, which are crucial to understand how the surface morphology of the heterogeneous system develops. In particular, the competition between the energetic effects, driving the system toward layer-by-layer growth, and the kinetic barriers, preventing diffusion and step-down motion from the terraces, is necessary to establish under which condition one specific growth mode can prevail. Calculations along these lines are currently in progress within the theoretical framework used for this investigation.

We thank J. Kirschner and J. Shen for helpful discussions. Calculations were performed on Cray computer of the German supercomputer center (HLRZ). This project was supported by Deutsche Forchungsgemeinschaft (DFG).
*Electronic address: stepan@valinux.physik.uni-halle.de

${ }^{1}$ B. Heinrich and J. F. Cochan, Adv. Phys. 42, 523 (1993).

${ }^{2}$ K. E. Johnson, D. D. Chambliss, R. J. Wilson, and S. Chiang, Surf. Sci. 313, L811 (1994).

${ }^{3}$ L. M. Falicov, D. T. Pierce, S. D. Bader, R. Gronsky, K. B. Hathaway, H. J. Hopster, D. N. Lambeth, S. P. Parkin, G. Prinz, M. Salamon, I. K. Schuller, and R. H. Victoria, J. Mater. Res. 5, 1299 (1990).

${ }^{4}$ J. Giergiel, J. Shen, J. Woltersdorf, A. Kirilyuk, and J. Kirschner, Phys. Rev. B 52, 8528 (1995).

${ }^{5}$ X. W. Zhou and H. N. G. Wadley, J. Appl. Phys. 84, 2301 (1998).

${ }^{6}$ B. Heinrich, J. F. Cochran, M. Kowalewski, J. Kirschner, Z. Celinski, A. S. Arrott, and K. Myrtle, Phys. Rev. B 44, 9348 (1991).

${ }^{7}$ P. Krams, F. Lauks, B. Hillebrands, and G. Güntherodt, Phys. Rev. Lett. 69, 3674 (1992).

${ }^{8}$ A. Cebollada, J. L. Martinez, J. M. Gallego, J. J. Miguel, R. Miranda, S. Ferrer, F. Batallan, G. Fillion, and J. P. Rebouillat, Phys. Rev. B 39, 9726 (1989).

${ }^{9}$ M. T. Johnson, S. T. Purcell, N. W. E. McGee, R. Coehoorn, Jaan de Stegge, and W. Hoving, Phys. Rev. Lett. 68, 2688 (1992).
${ }^{10}$ M. T. Kief and W. F. Egelhoff, Phys. Rev. B 47, 10785 (1993).

${ }^{11}$ J. Fassbender, R. Allenspach, and U. Dürig, Surf. Sci. 383, L742 (1997).

${ }^{12}$ F. R. de Boer, R. Boom, W. C. Mattens, A. Miedema, and A. K. Niessen, in Cohesion in Metals, edited by F. R. de Boer and G. Pettifor (North-Holland, Amsterdam, 1985), Vol. 1.

${ }^{13}$ H. Jenniches, M. Klaua, H. Hoche, and J. Kirschner, Appl. Phys. Lett. 69, 3339 (1996).

${ }^{14}$ F. Cleri and V. Rosato, Phys. Rev. B 48, 22 (1993).

${ }^{15}$ V. Rosato, B. Guillope, and B. Legrand, Philos. Mag. A 59, 321 (1989).

${ }^{16}$ K. Wildberger, V. S. Stepanyuk, P. Lang, R. Zeller, and P. H. Dederichs, Phys. Rev. Lett. 75, 509 (1995); V. S. Stepanyuk, W. Hergert, K. Wildberger, R. Zeller, and P. H. Dederichs, Phys. Rev. B 53, 2121 (1996); V. S. Stepanyuk, W. Hergert, P. Rennert, K. Wildberger, R. Zeller, and P. H. Dederichs, ibid. 54, 14 121 (1996).

${ }^{17}$ B. Drittler, M. Weinert, R. Zeller, and P. H. Dederichs, Phys. Rev. B 39, 930 (1989).

${ }^{18}$ T. Hoshino, W. Schweika, R. Zeller, and P. H. Dederichs, Phys. Rev. B 47, 5106 (1993). 
${ }^{19}$ N. Papanikolaou, R. Zeller, P. H. Dederichs, and N. Stefanou, Phys. Rev. B 55, 4157 (1997).

${ }^{20}$ J. R. Cerda, P. L. de Andres, A. Cebollada, R. Miranda, E. Navas, P. Schuster, C. M. Schneider, and J. Kirschner, J. Phys.: Condens. Matter 5, 2055 (1993).

${ }^{21}$ Very close values are obtained by comparing adsorption and embedding energy according to the definition adopted by J. Neugebauer and M. Scheffler [Phys. Rev. B 46, 16067 (1992)], which accounts for the formation energy of a vacancy in the substrate, the adsorption of an adatom on a vacancy site, and the displacement of the extracted surface atom at a surface step.

${ }^{22}$ P. H. B. Nonas, K. Wildberger, R. Zeller, and P. H. Dederichs, Phys. Rev. Lett. 80, 4574 (1998).

${ }^{23}$ G. Ehrlich and F. Hudda, J. Chem. Phys. 44, 1039 (1966); R. L. Schwoebel, J. Appl. Phys. 40, 614 (1969).

${ }^{24}$ C. G. Zimmermann, M. Yeadon, K. Nordlund, J. M. Gibson, R. S. Averback, U. Herr, and K. Samwer, Phys. Rev. Lett. 83, 1163 (1999). 